SUBJECT AREAS:

MECHANICAL

ENGINEERING

MATERIALS SCIENCE

Received

11 November 2013

Accepted

17 December 2013

Published

13 January 2014

Correspondence and requests for materials should be addressed to

T.-B.M. (mtb@mail.

tsinghua.edu.cn)

\section{OPEN A shear localization mechanism for lubricity of amorphous carbon materials}

\author{
Tian-Bao Ma' , Lin-Feng Wang' ${ }^{1}$ Yuan-Zhong Hu' ${ }^{1}$ Xin Li \& Hui Wang' \\ 'State Key Laboratory of Tribology, Tsinghua University, 100084 Beijing, China, ${ }^{2}$ Laser Micro/Nano Fabrication Laboratory, \\ School of Mechanical Engineering, Beijing Institute of Technology, 100081 Beijing, China.
}

Amorphous carbon is one of the most lubricious materials known, but the mechanism is not well understood. It is counterintuitive that such a strong covalent solid could exhibit exceptional lubricity. A prevailing view is that lubricity of amorphous carbon results from chemical passivation of dangling bonds on surfaces. Here we show instead that lubricity arises from shear induced strain localization, which, instead of homogeneous deformation, dominates the shearing process. Shear localization is characterized by covalent bond reorientation, phase transformation and structural ordering preferentially in a localized region, namely tribolayer, resulting in shear weakening. We further demonstrate an anomalous pressure induced transition from stick-slip friction to continuous sliding with ultralow friction, due to gradual clustering and layering of graphitic sheets in the tribolayer. The proposed shear localization mechanism sheds light on the mechanism of superlubricity, and would enrich our understanding of lubrication mechanism of a wide variety of amorphous materials.

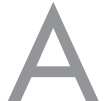

morphous carbon (a-C) is a covalent random network mostly composed of $\mathrm{sp}^{3}$ and $\mathrm{sp}^{2}$ hybridized sites without grain boundaries ${ }^{1}$, and is one of the most lubricious materials known, but the mechanism responsible for its lubricity is not well understood. It is counterintuitive that such a strong covalent solid could exhibit exceptional lubricity, and even superlubricity ${ }^{2}$. Lubricity of a-C film has been attributed to passivation of surface dangling bonds as well as to shear induced $\mathrm{sp}^{3} \rightarrow \mathrm{sp}^{2}$ rehybridization of the amorphous structure. Evidences exist to support both interpretations. By a hydrogen plasma treatment, the hydrogen-free a-C film was able to achieve very low and stable friction ${ }^{3}$, which was explained by the passivation of dangling $\sigma$ bonds by hydrogen atoms to form chemically inert surfaces. Similar mechanism has also been reported for the lubrication of a-C film by glycerol ${ }^{4}$ and ultrananocrystalline diamond (UNCD) by water molecules ${ }^{5}$. These observations are consistent with the passivation hypothesis. On the other hand, the lubricity may also result from $\mathrm{sp}^{3} \rightarrow \mathrm{sp}^{2}$ rehybridization, where $\mathrm{sp}^{2}$ phase is believed to be a softer carbon phase compared to $\mathrm{sp}^{3}$. The formation of $\mathrm{sp}^{2}$ dominated tribolayer has been observed in sliding interfaces between various carbon-based materials, including diamond ${ }^{6}, \mathrm{UNCD}^{5}$, diamond-like carbon ${ }^{7}$, amorphous carbon nitride ${ }^{8}$, and so on. Besides, shear induced graphitization mechanism was proposed to account for lubricity of a-C film ${ }^{9-14}$. The indentation and scratching induced graphitization of diamond was found under combined shear and hydrostatic compression stresses of up to $100 \mathrm{GPa}^{15}$. Most recently, definitive evidence for formation of graphitic tribolayer as a lubricating layer in artificial joints was provided by a combined characterization of electron energy loss spectra (EELS), high-resolution electron microscope (HREM) and spatially resolved Raman spectroscopy ${ }^{16}$ and by in situ TEM ${ }^{17,18}$, implying a conversion from disordered or amorphous carbon to a graphitic tribolayer during friction.

Due to the difficulties in direct observation of tribological processes by in-situ experiments with atomicscale resolution, it is not trivial to understand the contribution of each mechanism. However, molecular dynamics (MD) provides a powerful tool to capture atomic details and gain deeper understanding to the friction mechanism from the nanoscale. Here, we elucidate the microscopic processes underlying a-C friction using MD simulations. Sliding of a-C films against both diamond and self-mating a-C films leads to shear localization and phase transformation of a-C. Shear localization, in contrary with homogeneous deformation, is typical in shear deformation of amorphous materials, including granular media, metallic glasses, colloids and so on ${ }^{19,20}$, but has not been reported for a-C. Here, by using covalent bonding orientation angle for $\mathrm{C}-\mathrm{C}$ bonds as the prime signature, we characterize the evolution of shear band microstructure during sliding, and reveal its relevance with shear weakening to probe the physical origins of superlubricity. 
a

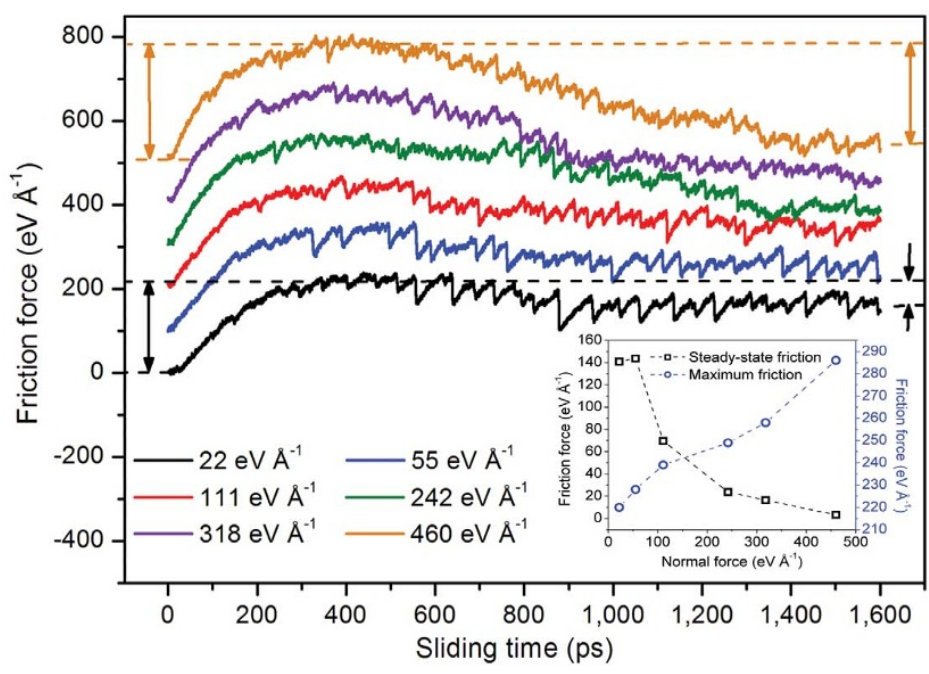

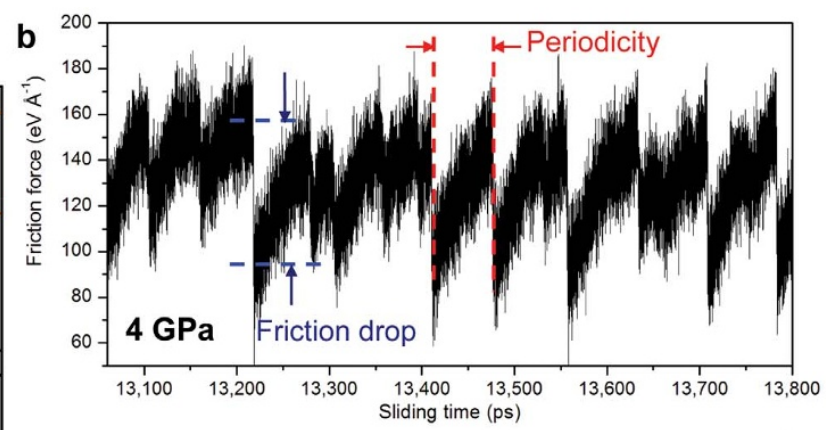

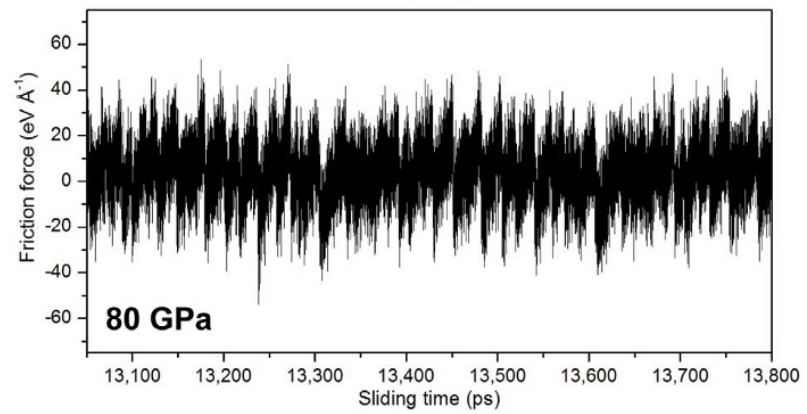

Figure 1 Shear weakening of a-C film. (a) Smoothed friction force plotted against sliding time. If not specified, the diamond/a-C film interfacial system with a sliding velocity of $10 \mathrm{~m} \mathrm{~s}^{-1}$ is used in this paper. The friction traces are shifted $100 \mathrm{eV} \AA^{-1}$ one by one with increasing pressure, in order to better distinguish between the traces. The left arrows show the maximum shear force of the a-C film. The right arrows show the magnitude of shear weakening. The black and yellow arrows are for the pressure of $4 \mathrm{GPa}$ and $80 \mathrm{GPa}$, respectively. The inset shows the relation between friction force and normal load. Both the averaged steady-state friction forces after shear weakening (black squares) and maximum friction forces before shear weakening (blue circles) are plotted. The dashed lines are plotted to guide the eyes. (b) Steady-state friction traces at 4 GPa and 80 GPa, showing stick-slip behaviors at $4 \mathrm{GPa}$. The friction drop and periodicity of the friction curve are depicted.

\section{Results}

During initial sliding, a-C film exhibits homogeneous shear deformation and onset of friction force. As shown in Fig. 1a, the friction force experiences a rapid increase during initial sliding followed by a gradual decrease at all normal pressures, showing occurrence of obvious shear weakening or running-in process. The maximum friction force increases monotonically with pressure, from roughly $220 \mathrm{eV}^{-1}$ at $22 \mathrm{eV} \AA^{-1}$ normal force (corresponding to a normal pressure of about $4 \mathrm{GPa}$ ) to $270 \mathrm{eV} \AA^{-1}$ at $460 \mathrm{eV} \AA^{-1}$ normal force $(80 \mathrm{GPa})$. On the other hand, shear weakening, which is designated by the friction drop from maximum to steady-state friction, becomes much more pronounced at higher pressures. The steady-state friction decreases monotonically from $140 \mathrm{eV} \AA^{-1}$ to $3 \mathrm{eV}^{-1}$ with increasing pressure. The inset in Fig. 1a shows different friction-load dependencies in two sliding regimes: the friction force increases with normal load before shear weakening, while the steady-state friction force after shear weakening decreases with normal load. Serrated-type stick-slip friction is another important feature of the friction traces, especially at lower pressures. The stick-slip has relatively irregular amplitude of friction drop and periodicity, but they do show some dependence on pressure: both decrease with increasing pressure. This could be observed clearly by the steadystate friction traces in Fig. 1b. The friction drop at $4 \mathrm{GPa}$ could reach a maximum of $80 \mathrm{eV} \AA^{-1}$ with periodicities of several tens to 100 ps with an average friction of about $130 \mathrm{eV} \AA^{-1}$, while the friction trace at $80 \mathrm{GPa}$ only exhibits oscillations around zero with periodicities of about 10 ps and nearly vanishing stick-slip, resulting in a nearfrictionless state with little energy dissipation.

In order to reveal the mechanism for shear weakening, it is necessary to correlate a certain structural feature with shear force and phase transformation. We propose the $\mathrm{C}-\mathrm{C}$ covalent bonding orientation angle (CBOA) as an appropriate monitoring parameter, due to the directionality of covalent bonds. The CBOA is defined as the angle between $\mathrm{C}-\mathrm{C}$ bond and the $\mathrm{x}-\mathrm{y}$ plane. For diamond (100) surface the $\mathrm{CBOA}$ is $35.2^{\circ}$, and for as-deposited a-C film the average $\mathrm{CBOA}$ is calculated to be around $35^{\circ}$ homogeneously throughout the film. After compression at $4 \mathrm{GPa}$ and $80 \mathrm{GPa}$, the CBOA decreases to about $34^{\circ}$ and $31^{\circ}$, respectively. However after shear at $80 \mathrm{GPa}$ for $10 \mathrm{~ns}$, a significant decrease of CBOA occurs in the region from layer A to $G$ with a minimum averaged value of $7.6^{\circ}$ at layer $C$, as shown in Figs. $2 \mathrm{a}$ and $2 \mathrm{~b}$. Meanwhile, a much smaller decrease of the CBOA is also observed in other parts of the film (for example, region between layers $\mathrm{G}$ and $\mathrm{H}$ ). Similar structural feature is observed at $4 \mathrm{GPa}$ in Fig. $2 \mathrm{c}$ and 2 d. So a-C does not exhibit homogeneous shear deformation with Newtonian fluid-like behavior throughout the material, but rather exhibit localized deformation in a fashion of shear band (see Supplementary Movies S1 and S2). It is found that a thinner shear band with sharper boundary is formed showing a larger strain rate within the shear band at $80 \mathrm{GPa}$. The ultralow friction in this case is due to the formation of sliding plane between graphene-like layers as shown in Fig. 2a and Fig. S2a. The interlayer interaction is further verified by density functional theory (DFT) calculations in Supplementary Information S-II. The large load bearing capacity results from the $\pi$ orbital electrons interaction, while the low shear strength results from the absence of interlayer $\sigma$ bonds and the incommensurate geometry (Fig. S2b). The normal pressure and interlayer distance for the graphene-like layers agree quite well with that of the transition state of both compression induced graphite-todiamond transition ${ }^{21,22}$ and shear induced diamond-to-graphite transition ${ }^{23}$ by DFT calculations, which is believed to be the energetically favored pathway for the transition to happen under high pressure and shear and explains the mechanism of initial nucleation of local graphene-like structures during a-C sliding.

The localized shear deformation provides a quantitative description of the velocity accommodation mode ${ }^{24}$ for covalently bonded solids from the atomic scale. It should be noted that as a-C film is grown homogeneously without voids, the localization or shear band seems to generate near the initial contacting interface at low 
$80 \mathrm{GPa}$
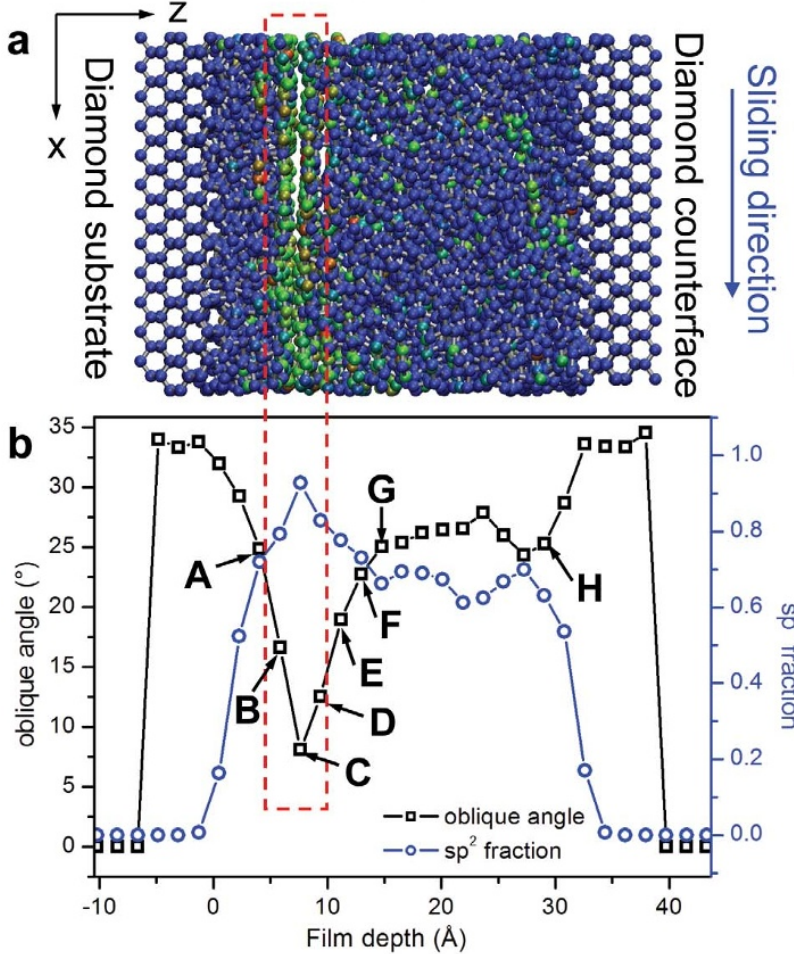

$4 \mathrm{GPa}$

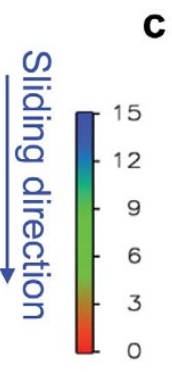

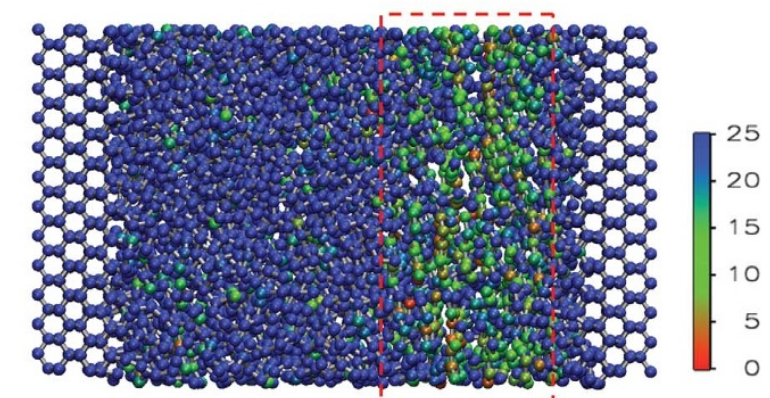

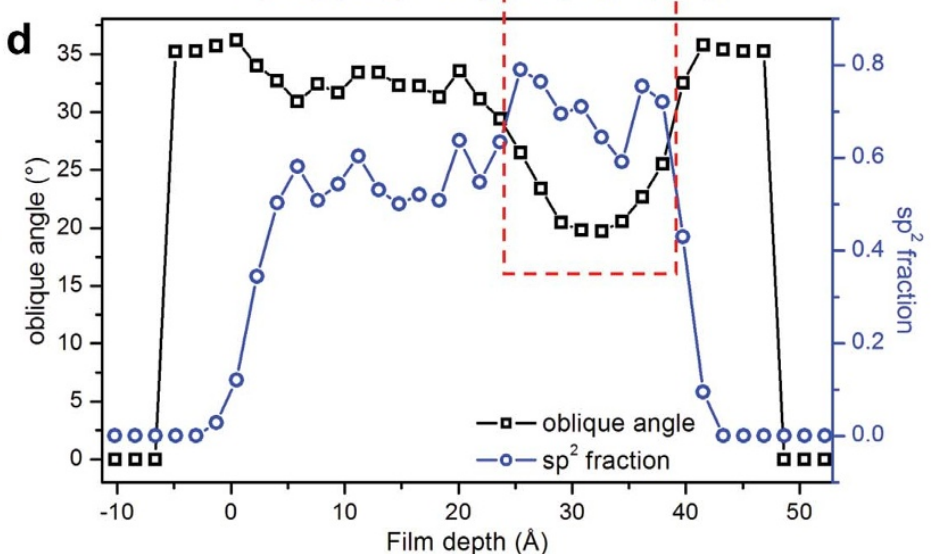

Figure $2 \mid$ Shear localization and structural transformation of a-C film for $80 \mathrm{GPa}$ (left) and $4 \mathrm{GPa}$ (right). (a, c) System snapshots in steady-state friction stage at $\mathrm{t}=10 \mathrm{~ns}$, color for each atom represents quantitative value of the averaged CBOA of all C-C bonds connecting the specific atom with its nearest neighbors. The RGB color coding is shown. Note that the upper scale value is different for the two pressures, in order to signify different degree of shear localization. (b, d) Depth profiles of averaged CBOA and sp $\mathrm{p}^{2}$ fraction. The values are averaged in bins with equal thickness of $1.78 \AA$ at $\mathrm{t}=10 \mathrm{~ns}$. The dashed rectangle denotes the position of the shear bands. Layers A to $\mathrm{H}$ are depicted for detailed analysis in Fig. 3 and discussion in the text.

pressures where a large amount of defects or free volume $e^{25,26}$ exists. However, the localization position in the film seems unpredictable at high pressures, which originates from the local strain fluctuations during shear and is susceptibly affected by pressure, sliding velocity, temperature and pairing materials. Graphitization, as a special form of shear localization of a- $\mathrm{C}^{27}$, gives it distinctive lubricity from other amorphous materials.

The phase transformation is characterized by $\mathrm{sp}^{3} \rightarrow \mathrm{sp}^{2}$ rehybridization. The $\mathrm{sp}^{2}$ fraction in a-C before shear is around $38 \%$ homogeneously throughout the film (the remainder $62 \%$ mostly $\mathrm{sp}^{3}$ hybridized), which increases during shear at all pressures. A remarkable mirror symmetry is observed for CBOA and $\mathrm{sp}^{2}$ fraction profiles at both pressures from Figs. $2 \mathrm{~b}$ and $2 \mathrm{~d}$. The increase of $\mathrm{sp}^{2}$ fraction is more marked in the shear localization region, with a maximum of $93 \%$ and $80 \%$ at $80 \mathrm{GPa}$ and $4 \mathrm{GPa}$, respectively, although $\mathrm{sp}^{2}$ fraction also increase in other parts of the film to around $65 \%$ and $55 \%$ at $80 \mathrm{GPa}$ and $4 \mathrm{GPa}$ respectively.

The evolution of shear localization and phase transformation is shown in Fig. 3, where the variation of CBOA and $\mathrm{sp}^{2}$ fraction of each layer as depicted in Fig. $2 \mathrm{~b}$ is traced at $80 \mathrm{GPa}$. During the first $600 \mathrm{ps}$ of the shear process, all these layers experience similar deformation together as shown by the coincidence of CBOA traces. However, layer A, G, H stops further shear deformation after 600 ps (the $\mathrm{CBOA}$ reaches a stable value) as the shear is localized in the region from $\mathrm{B}$ to $\mathrm{F}$. And $\mathrm{C}$ is the last layer to reach stable CBOA, after which the shear force drops to nearly zero and sliding happens between the graphene-like layers. The evolution of CBOA after localization initiates is consistent with the weakening stage of friction trace after 600 ps in Fig. 1a, which highlights the relationship between shear localization and lubricity of a-C. The physical meaning of a small CBOA is a predominant orientation of $\mathrm{C}-\mathrm{C}$ covalent bonds along (not perpendicular to) the sliding direction, corresponding to a structure feature conformable (not resistant) to shear. It is also inspiring to find that $\mathrm{sp}^{2}$ fraction traces show graceful mirror symmetry with CBOA traces, showing strong correlation between $\mathrm{sp}^{3} \rightarrow \mathrm{sp}^{2}$ phase transformation and shear deformation. Further increase in $\mathrm{sp}^{2}$ fraction is observed in the shear band after shear localization initiates. However, by comparing Figs. $3 \mathrm{a}$ and $3 \mathrm{~b}, \mathrm{sp}^{2}$ fraction has already increased dramatically from less than $40 \%$ to nearly $70 \%$ within first 600 ps before shear localization initiates, while no obvious shear weakening occurs during this period as shown in Fig. 1a. So the rehybridization happens mainly during the homogeneous shear deformation before localization at both $80 \mathrm{GPa}$ and $4 \mathrm{GPa}$ (see the obvious atomic color changes during this stage in Supplementary Movies S3 and S4). The $\mathrm{sp}^{3} \rightarrow \mathrm{sp}^{2}$ rehybridization of a-C film during friction has been detected by extensive Raman analysis ${ }^{7}$, which was postulated to be the mechanism of a-C lubricity. However, our simulation results show that simply $\mathrm{sp}^{3} \rightarrow \mathrm{sp}^{2}$ rehybridization and amorphization does not consequentially lead to lubricity. Ultralow friction could only be achieved collectively by both highly shear localization (very low $\mathrm{CBOA}$, say less than $10^{\circ}$ ) and highly phase transformation (very high $\mathrm{sp}^{2}$ fraction, say more than $90 \%$ ) in the very thin localized layer. It could be found by comparing Fig. $2 \mathrm{~b}$ and Fig. $2 \mathrm{~d}$ that CBOA is the key parameter to distinguish high and low friction (with a value of $20^{\circ}$ and $7.6^{\circ}$ at $4 \mathrm{GPa}$ and $80 \mathrm{GPa}$ respectively), which is an important representation of the degree of structural ordering, graphitic clustering and shear strength of the tribolayer.

Pressure plays a dominant role in shear localization and structural transformation. At low normal pressure, a-C is less compressed with a less compact structure, allowing flexible responses to shearing, and specifically, more freedom for atomic movement in film depth (z) direction. This could be inferred from Fig. $4 \mathrm{a}$ and $4 \mathrm{~b}$, where the trace 

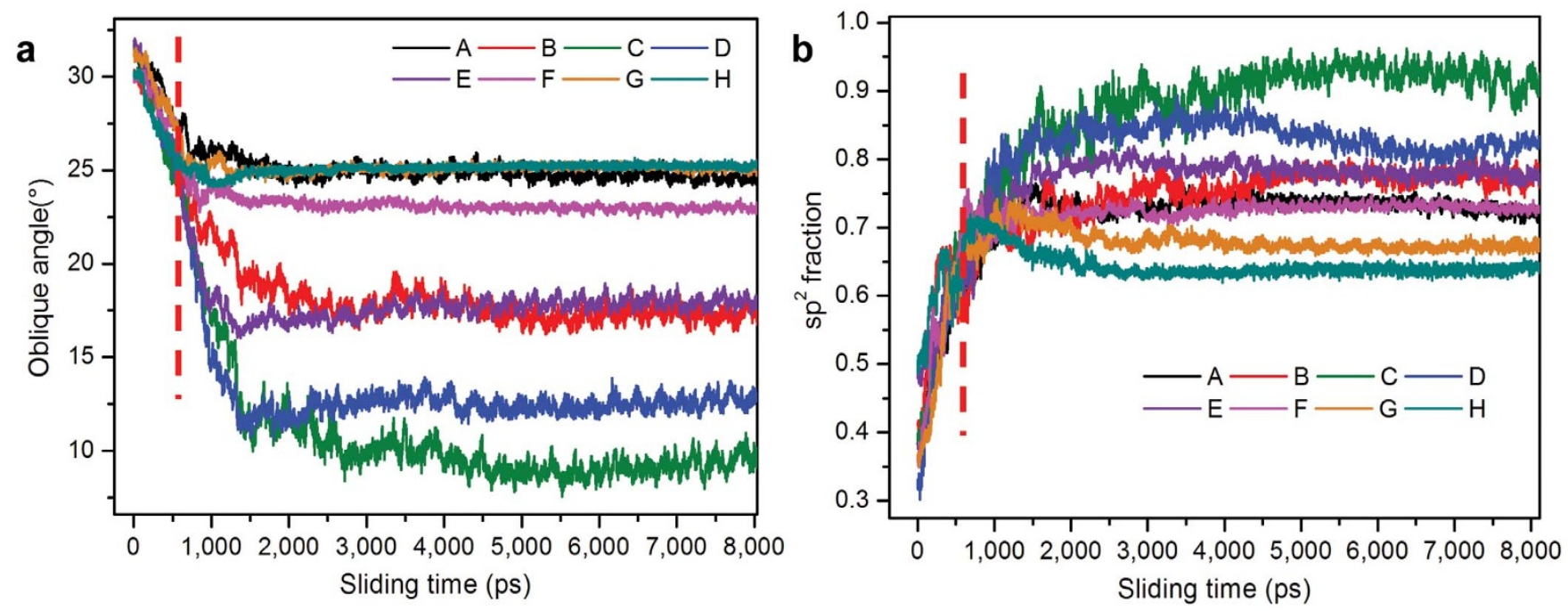

Figure 3 Time evolution of deformation and phase transformation during friction at a pressure of $80 \mathrm{GPa}$. (a) CBOA variations, showing inhomogeneous shear deformation of the film. From A to H, each curve shows the averaged CBOA corresponding to a layer shown in Fig. $2 \mathrm{~b}$. (b) sp ${ }^{2}$ fraction variations in layer A to $\mathrm{H}$, showing gradient of phase transformation along the film depth direction. The vertical dashed lines show the initiation of shear localization at 600 ps.

of film thickness shows large fluctuations in different wavelengths. The film thickness decreases at first when the surfaces originally in partial contact are seeking more compliant contacting geometry. Then the film thickness increases due to a shear induced volumetric dilation and free volume generation. Interestingly, the friction and film thickness traces in both wavelengths (as depicted by the fast fourier transform (FFT) smoothed traces in Fig. 4a and local enlargement in Fig. 4b) exhibit mirror symmetry. The volumetric change allows atoms in the shear band undergo approach-separation movements, leading to stick-slip behaviors. As clearly shown in Fig. 4b, each slip process corresponds to a sudden increase of film thickness, which is analogous to the slip-induced dilation observed in granular material $s^{28}$. As an amorphous solid, a-C does not show the characteristic atomic lattice stick-slip behavior for crystalline materials ${ }^{29}$. It should also be distinguished from the shear-melting and recrystallization cycle in molecularly thin confined liquid films $\mathrm{s}^{30,31}$. The stickslip friction observed here is rather elastic segment-plastic event cycles $^{32}$ caused by collective covalent bond forming and breaking in the shear band, which is similar to that in shear of metallic glasses representing a full cycle of shear-band nucleation, propagation and arrest $^{33}$.

On the contrary, $\mathrm{a}-\mathrm{C}$ at high pressure has a highly compact and stiff structure, restricting the movement of carbon atoms in $\mathrm{z}$ direction and prohibiting shear dilation and generation of free volume. The film thickness trace is much smoother, while the symmetry between friction and film thickness traces is broken (Fig. 4c). The restrained movement of carbon atoms is favored in $x-y$ plane under shear, which accounts for the reorganization of atoms in sliding direction and formation of graphene-like layers. At intermediate pressures, enhanced shear localization (characterized by decreasing CBOA), structural ordering and clustering of graphitic sheets with lower friction and weaker stick-slip are observed with increasing pressures, until graphene-like layering is formed. The promotion of sliding induced graphitization of a-C films by increasing normal pressure was also observed experimentally ${ }^{9}$. In a previous study, a transition from atomic lattice stick-slip to continuous sliding was observed by decreasing the normal load, where the dominating factor is the decreasing potential energy corrugation rather than stiffness, because the atomic structure of the contact does not change in the applied load range ${ }^{34}$. However in the present system, not only does the steady-state shear strength decrease (corresponding to a less corrugated potential energy surface), but also the system stiffness increase with increasing pressure. This explains the variation of stick-slip periodicity and amplitude with applied normal pressure, namely with higher pressure, the system is less compliant, so the stick-slip is less obvious. Interestingly, a high pressure lubricity has been recently reported for confined Lennard-Jones lubricant film ${ }^{35}$. The friction decrease of lubricant confined between crystalline sliders with increasing pressure is attributed to two factors: (1) High friction with bulk lubricant shear, easier at low pressure and low
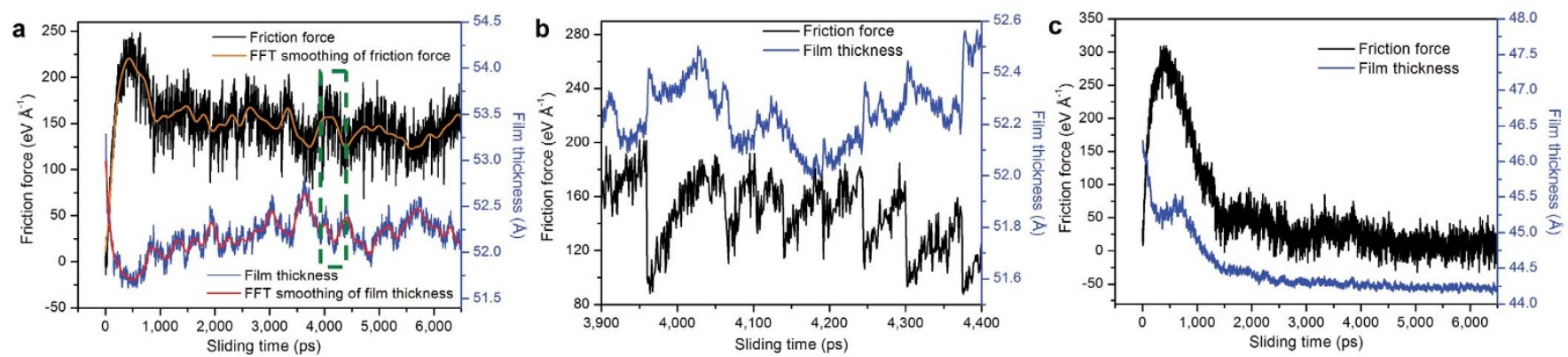

Figure $4 \mid$ Variations of friction and film thickness with sliding time at normal pressures of (a) 4 GPa and (c) 80 GPa. The film thickness here is estimated by the distance between the lower and upper rigid layers, which is a good approximation of the a-C film thickness. (a) The FFT smoothing of friction and film thickness traces is conducted for observation of good correlation between the two traces in larger wavelength. (b) Enlargement of the time period between 3900 ps- 4400 ps as shown by the green rectangle in Fig. 4a. 
density, could disappear after relayering when density is higher; (2) Formation of incommensurate slider-lubricant interfacial sliding interface ${ }^{35}$. It should be mentioned that lubricity of materials in sliding contact has also been attributed to velocity weakening ${ }^{36}$, flash heating or melting ${ }^{37,38}$. So in separated simulations, a strain rate study has been carried out over a wide range of applied velocity from $1 \mathrm{~m} \mathrm{~s}^{-1}$ to $100 \mathrm{~m} \mathrm{~s}^{-1}$. Over this velocity range, we found that the main conclusions about shear localization and phase transformation still hold, shear weakening and transition from stick-slip to continuous sliding by increasing the pressure still exist, the formation of very thin localized graphene-like tribolayer with ultralow friction is still observed at a velocity of $1 \mathrm{~m} \mathrm{~s}^{-1}$. Although slight strain rate induced weakening is also observed, i.e. the friction is slightly smaller at a higher strain rate, the effect is much less pronounced than pressure induced weakening. So the main findings here are not limited to very high shear rate regime.

\section{Discussion}

In summary, shear weakening and transition from stick-slip friction to continuous sliding of $\mathrm{a}-\mathrm{C}$ film by increasing pressure are observed by $\mathrm{MD}$ simulations. The $\mathrm{a}-\mathrm{C}$ film experiences steady-state localized deformation rather than homogeneous fluid-like deformation under shear. The shear localization or formation of tribolayer of a-C is characterized by structural ordering with $\mathrm{C}-\mathrm{C}$ bond reorientation towards the sliding direction, clustering of graphene-like sheets, which explains the physical origin of the lubricity of a-C materials. The ultralow friction at high pressure is due to the formation of sliding plane between graphene-like layers. Although a scenario of graphitized material formation on surface asperities of a-C was speculated as the reason for superlubricity of a- $\mathrm{C}^{29}$, no direct theoretical results have been reported to prove the speculation. Recently superlubricity of graphite or graphene has become a topic which arouses widespread interest and has great scientific and potential technological values ${ }^{39,40}$. However, to ensure persistent incommensurability during sliding of graphene remains a technical challenge to tackle in order to achieve superlubricity in practice $e^{41,42}$. The present study shows that incommensurate graphene-like layers in stable sliding could be ready to form by simply rubbing two a-C asperities, which is also in line with the above mentioned speculation ${ }^{29}$.

The question of whether the primary lubrication mechanism of a$\mathrm{C}$ is surface passivation or shear localization is still open and needs further experimental verifications with atomic-scale resolution. However, we have demonstrated that shear localization is a viable and important lubrication mechanism of a-C, which sheds light on some heretofore ambiguous concepts in superlubricity. For example, all simulations show obvious $\mathrm{sp}^{3} \rightarrow \mathrm{sp}^{2}$ rehybridization under shear, yet with a wide range of steady-state friction. So simply rehybridization cannot guarantee a superlow friction. On the other hand, hydrogen passivation alone may also not well explain the origin of superlubricity, since hydrogen atoms are not able to totally prevent covalent bond formation between hydrogenated a-C surfaces ${ }^{43}$, especially in situations where dissociation and removal of surface termination occurs during the rubbing processes ${ }^{9,44}$. The proposed shear localization mechanism would enrich our understanding of lubrication mechanism of a wide variety of amorphous materials.

\section{Methods}

Molecular dynamics (MD) simulation was carried out for friction behaviors of amorphous carbon (a-C) films. The a-C films were deposited atom-by-atom with a thickness of about $4 \mathrm{~nm}^{45}$, with the lateral cell dimensions of $3 \mathrm{~nm} \times 3 \mathrm{~nm}$. Both diamond (100) reconstructed surface and self-mated a-C surface were used as the mating materials, resulting in system sizes of 7191 and 12078 atoms respectively. The large system size is necessary for simulating plastic deformation process, such as localized shear band formation in a-C, which is difficult to learn otherwise by density functional theory (DFT) calculations because of the huge computational cost. The choice of the system size is a trade-off between accuracy and cost, for the reactive forcefield adopted here is computationally expensive. The clean carbon surfaces were used here under the assumption of dissociation and removal of any surface contaminations or termination during initial sliding processes ${ }^{44}$. The sliding process is accomplished using a scheme similar to previous studies ${ }^{43,46}$. The bottom and top two atomic layers of the system were held rigid, and the atoms in neighboring three layers were coupled to a Langevin thermostat to keep at a constant temperature at $300 \mathrm{~K}$. The second generation of reactive empirical bond-order (REBO) potential ${ }^{47}$ with an increased cutoff ${ }^{48}$ was adopted to describe the $\mathrm{C}-\mathrm{C}$ interatomic interactions, which is able to model covalent bond forming and breaking, rehybridization, chemical reactions and tribological processes. By a separate simulation (see Supplementary Information S-I) of liquid quenching of an a-C sample similar to previous studies $^{49}$, the density-sp ${ }^{3}$ fraction relationship was well reproduced compared to $\mathrm{DFT}^{50}$ and nonorthogonal tight-binding (NOTB) ${ }^{51}$ calculations, which verifies the validity of the potential under high pressures. Also the a-C film structure in atom-by-atom deposition simulations using REBO potential also shows good agreements with experimental results ${ }^{45,48}$. The normal pressures adopted in this study range from $500 \mathrm{MPa}$ to $80 \mathrm{GPa}$, which basically cover the real pressure range in a-C asperity contacts. High pressure could be possible during instantaneous contact of asperities during friction, which may be orders of magnitude higher than the nominal contact pressure estimated in experiments. The sliding velocity in most calculations ranges from $10 \mathrm{~m} \mathrm{~s}^{-1}$ to $100 \mathrm{~m} \mathrm{~s}^{-1}$ (also a couple of cases with $1 \mathrm{~m} \mathrm{~s}^{-1}$ for verification). If not specified, the sliding velocity is $10 \mathrm{~m} \mathrm{~s}^{-1}$ and the sliding simulation lasts $20 \mathrm{~ns}$ in order to get steady-state friction in this paper.

The DFT calculation was conducted using Vienna Ab initio Simulation Package $(V A S P)^{52}$. The exchange-correlation functional was represented with the GGA in the form of the Perdew-Burke-Ernzerhof $(\mathrm{PBE})^{53}$ functional. The projector-augmented wave $(\mathrm{PAW})^{54}$ method was used to describe the interaction of valence electrons with atomic cores. The cutoff energy was $400 \mathrm{eV}$. The $k$ points were generated through the Monkhorst-Pack (MP) algorithm ${ }^{55}$. The integration of the Brillouin zone was performed using a $3 \times 3 \times 1 \mathrm{MP}$ grid and tetrahedron smearing method.

1. Robertson, J. Diamond-like amorphous carbon. Mater. Sci. Eng. R 37, 129-281 (2002).

2. Erdemir, A. \& Donnet, C. Tribology of diamond-like carbon films: recent progress and future prospects. J. Phys. D: Appl. Phys. 39, R311-R327 (2006).

3. Eryilmaz, O. L. \& Erdemir, A. On the hydrogen lubrication mechanism(s) of DLC films: An imaging TOF-SIMS study. Surf. Coat. Technol. 203, 750-755 (2008).

4. Matta, C. et al. Superlubricity and tribochemistry of polyhydric alcohols. Phys. Rev. B 78, 085436 (2008).

5. Konicek, A. R. et al. Origin of ultralow friction and wear in ultrananocrystalline diamond. Phys. Rev. Lett. 100, 235502 (2008).

6. Pastewka, L., Moser, S., Gumbsch, P. \& Moseler, M. Anisotropic mechanical amorphization drives wear in diamond. Nat. Mater. 10, 34-38 (2011).

7. Sanchez-Lopez, J. C., Erdemir, A., Donnet, C. \& Rojas, T. C. Friction-induced structural transformations of diamondlike carbon coatings under various atmospheres. Surf. Coat. Technol. 163-164, 444-450 (2003).

8. Wang, P., Hirose, M., Suzuki, Y. \& Adachi, K. Carbon tribo-layer for super-low friction of amorphous carbon nitride coatings in inert gas environments. Surf. Coat. Technol. 221,163-172 (2013).

9. Liu, Y., Erdemir, A. \& Meletis, E. I. An investigation of the relationship between graphitization and frictional behavior of DLC coatings. Surf. Coat. Technol. 8687, 564-568 (1996).

10. Ma, T. B., Hu, Y. Z. \& Wang, H. Molecular dynamics simulation of shear-induced graphitization of amorphous carbon films. Carbon 47, 1953-1957 (2009).

11. Ma, T. B., Hu, Y. Z., Xu, L., Wang, L. F. \& Wang, H. Shear-induced lamellar ordering and interfacial sliding in amorphous carbon films: A superlow friction regime. Chem. Phys. Lett. 514, 325-329 (2011).

12. Wang, Y. X. et al. Nanocomposite microstructure and environment self-adapted tribological properties of highly hard graphite-like film. Tribol. Lett. 40, 301-310 (2010).

13. Tasdemir, H. A. et al. Wear behaviour of tetrahedral amorphous diamond-like carbon (ta-C DLC) in additive containing lubricants. Wear 307, 1-9 (2013).

14. Lei, B. L. et al. New insights into the microstructure of the friction surface layer of C/C composites. Carbon 49, 4554-4562 (2011).

15. Gogotsi, Y. G., Kailer, A. \& Nickel, K. G. Transformation of diamond to graphite. Nature 401, 663-664 (1999).

16. Liao, Y. et al. Graphitic tribological layers in metal-on-metal hip replacements. Science 334, 1687-1690 (2011).

17. Merkle, A. P., Erdemir, A., Eryilmaz, O. L., Johnson, J. A. \& Marks, L. D. In situ TEM studies of tribo-induced bonding modifications in near-frictionless carbon films. Carbon 48, 587-591 (2010).

18. Merkle, A. P. Tribological interfaces studied by an analytical dislocation model and in-situ transmission electron microscopy. Ph.D. Thesis. Northwestern University, AAI3251922 (2007).

19. Daub, E. G. \& Carlson, J. M. Stick-slip instabilities and shear strain localization in amorphous materials. Phys. Rev. E 80, 066113 (2009).

20. Greer, A. L., Cheng, Y. Q. \& Ma, E. Shear bands in metallic glasses. Mater. Sci. Eng. $R$ 74, 71-132 (2013).

21. Fahy, S., Louie, S. G. \& Cohen, M. L. Pseudopotential total-energy study of the transition from rhombohedral graphite to diamond. Phys. Rev. B 34, 1191-1199 (1986). 
22. Khaliullin, R. Z., Eshet, H. T., Kühne, D., Behler, J. \& Parrinello, M. Nucleation mechanism for the direct graphite-to-diamond phase transition. Nat. Mater. 10, 693-697 (2011).

23. Chacham, H. \& Kleinman, L. Instabilities in diamond under high shear stress. Phys. Rev. Lett. 85, 4904-4907 (2000).

24. Chromik, R. R., Winfrey, A. L., Lüning, J., Nemanich, R. J. \& Wahl, K. J. Run-in behavior of nanocrystalline diamond coatings studied by in situ tribometry. Wear 265, 477-489 (2008).

25. Cohen, M. \& Turnbull, D. Molecular transport in liquids and glasses. J. Chem. Phys. 31, 1164-1169 (1959).

26. Shi, Y. F., Katz, M. B., Li, H. \& Falk, M. L. Evaluation of the disorder temperature and free-volume formalisms via simulations of shear banding in amorphous solids. Phys. Rev. Lett. 98, 185505 (2007).

27. Oohashi, K., Hirose, T. \& Shimamoto, T. Shear-induced graphitization of carbonaceous materials during seismic fault motion: Experiments and possible implications for fault mechanics. J. Struct. Geol. 33, 1122-1134 (2011).

28. Nasuno, S., Kudrolli, A., Bak, A. \& Gollub, J. P. Time-resolved studies of stick-slip friction in sheared granular layers. Phys. Rev. E 58, 2161-2171 (1998).

29. Dienwiebel, M. et al. Superlubricity of graphite. Phys. Rev. Lett. 92, 126101 (2004).

30. Thompson, P. A. \& Robbins, M. O. Origin of stick-slip motion in boundary lubrication. Science 250, 792-794 (1990).

31. Gourdon, D. \& Israelachvili, J. N. Transitions between smooth and complex stickslip sliding of surfaces. Phys. Rev. E 68, 021602 (2003).

32. Karmakar, S., Lemaître, A., Lerner, E. \& Procaccia, I. Predicting plastic flow events in athermal shear-strained amorphous solids. Phys. Rev. Lett. 104, 215502 (2010).

33. Klaumünzer, D., Maaß, R. \& Löffler, J. F. Stick-slip dynamics and recent insights into shear banding in metallic glasses. J. Mater. Res. 26, 1453-1463 (2011).

34. Socoliuc, A., Bennewitz, R., Gnecco, E. \& Meyer, E. Transition from stick-slip to continuous sliding in atomic friction: Entering a new regime of ultralow friction. Phys. Rev. Lett. 92, 134301 (2004).

35. Vanossi, A., Benassi, A., Varini, N. \& Tosatti, E. High-pressure lubricity at the meso- and nanoscale. Phys. Rev. B 87, 045412 (2013).

36. Braun, O. M. \& Röder, J. Transition from stick-slip to smooth sliding: an earthquakelike model. Phys. Rev. Lett. 88, 096102 (2002).

37. Dawson, B. D., Lee, S. M. \& Krim, J. Tribo-induced melting transition at a sliding asperity contact. Phys. Rev. Lett. 103, 205502 (2009).

38. Goldsby, D. L. \& Tullis, T. E. Flash heating leads to low frictional strength of crustal rocks at earthquake slip rates. Science 334, 216-218 (2011).

39. Yang, J. R. et al. Observation of high-speed microscale superlubricity in graphite. Phys. Rev. Lett. 110, 255504 (2013)

40. Lee, C. et al. Frictional characteristics of atomically thin sheets. Science 328, 76-80 (2010).

41. Filippov, A. E., Dienwiebel, M., Frenken, J. W. M., Klafter, J. \& Urbakh, M. Torque and twist against superlubricity. Phys. Rev. Lett. 100, 046102 (2008).

42. Hirano, M., Shinjo, K., Kaneko, R. \& Murata, Y. Observation of superlubricity by scanning tunneling microscopy. Phys. Rev. Lett. 78, 1448-1451 (1997)

43. Schall, J. D., Gao, G. T. \& Harrison, J. A. Effects of adhesion and transfer film formation on the tribology of self-mated DLC contacts. J. Phys. Chem. C 114, 5321-5330 (2010).

44. Harrison, J. A. \& Brenner, D. W. Simulated tribochemistry: An atomic-scale view of the wear of diamond. J. Am. Chem. Soc. 116, 10399-10402 (1994).

45. Ma, T. B., Hu, Y. Z., Wang, H. \& Li, X. Microstructural and stress properties of ultrathin diamondlike carbon films during growth: Molecular dynamics simulations. Phys. Rev. B 75, 035425 (2007).
46. Pastewka, L., Moser, S. \& Moseler, M. Atomistic insights into the running-in, lubrication, and failure of hydrogenated diamond-like carbon coatings. Tribol. Lett. 39, 49-61 (2010).

47. Brenner, D. W. et al. A second-generation reactive empirical bond order (REBO) potential energy expression for hydrocarbons. J. Phys.: Condens. Matter 14,783-802 (2002).

48. Jäger, H. U. \& Belov, A. Y. ta-C deposition simulations: Film properties and timeresolved dynamics of film formation. Phys. Rev. B 68, 024201 (2003).

49. Pastewka, L., Pou, P., Pérez, R., Gumbsch, P. \& Moseler, M. Describing bondbreaking processes by reactive potentials: Importance of an environmentdependent interaction range. Phys. Rev. B 78, 161402(R) (2008).

50. McCulloch, D. G., McKenzie, D. R. \& Goringe, C. M. Ab initio simulations of the structure of amorphous carbon. Phys. Rev. B 61, 2349-2355 (2000).

51. Marks, N. A. et al. Comparison of density-functional, tight-binding, and empirical methods for the simulation of amorphous carbon. Phys. Rev. B 65, 075411 (2000).

52. Kresse, G. \& Hafner, J. Ab initio molecular-dynamics simulation of the liquidmetal-amorphous-semiconductor transition in germanium. Phys. Rev. B 49, 14251-14269 (1994).

53. Perdew, J. P., Burke, K. \& Ernzerhof, M. Generalized Gradient Approximation Made Simple. Phys. Rev. Lett. 77, 3865-3868 (1996).

54. Blöchl, P. E. Projector augmented-wave method. Phys. Rev. B 50, 17953-17979 (1994).

55. Monkhorst, H. J. \& Pack, J. D. Special points for brillouin-zone integrations. Phys. Rev. B 13, 5188-5192 (1976).

\section{Acknowledgments}

This work is financially supported by the National Natural Science Foundation of China (Grant Nos. 51375010, 51075226, 51321092). Computations were carried out on the Tsinghua National Laboratory for Information Science and Technology.

\section{Author contributions}

T.M., Y.H. and X.L. conceived the molecular dynamics simulation and T.M. carried it out L.W. carried out the density functional theory calculations. T.M., X.L. and H.W. designed the data analysis and T.M. and L.W. carried it out. All authors discussed the results and wrote the paper.

\section{Additional information}

Supplementary information accompanies this paper at http://www.nature.com/ scientificreports

Competing financial interests: The authors declare no competing financial interests. How to cite this article: Ma, T.-B., Wang, L.-F., Hu, Y.-Z., Li, X. \& Wang, H. A shear localization mechanism for lubricity of amorphous carbon materials. Sci. Rep. 4, 3662; DOI:10.1038/srep03662 (2014)

cc)(-) $\Theta$ This work is licensed under a Creative Commons AttributionBY No No NonCommercial-NoDerivs 3.0 Unported license. To view a copy of this license, visit http://creativecommons.org/licenses/by-nc-nd/3.0 